\title{
Ischaemic complications of graduated compression stockings in the treatment of deep venous thrombosis
}

\author{
Neil D. Merrett and Kevin C. Hanel \\ St George Hospital, Sydney, New South Wales, Australia
}

\begin{abstract}
Summary: Graduated compression stockings are frequently used in the prevention of deep venous thrombosis and the treatment of venous insufficiency. Two patients are discussed who sustained ischaemic complications after application of graduated compression stockings. Review of the literature demonstrates that low cutaneous pressures significantly decrease local blood flow and that the amount of pressure exerted by graduated compression stockings increases significantly with increases in leg girth. Ischaemic complications associated with the use of these stockings also appears to be more common than previously thought and any policy of routine prescription to patients should be questioned.
\end{abstract}

\section{Introduction}

Graduated compression stockings have become commonplace as their efficacy in the prevention of post-operative deep venous thrombosis (DVT) has come to be appreciated. Multiple trials have demonstrated a significant reduction in the incidence of post-operative DVT as compared with placebo, ${ }^{1-3}$ and a similar efficacy to that of other regimes., The compressive effects of the stockings have also been widely used in the treatment of established DVT.

Graduated compression stockings have in the past been described as being relatively free of side effects $^{2,4}$ with the most frequently described problems being those of heat and difficulty in keeping them up. The manufacturers do, however, caution against their use in patients with severe atherosclerosis and ischaemic ulceration. This efficacy in the prevention of post-operative DVT, the simplicity of their use and the apparent lack of side effects has made them so popular that they are now being used routinely in many units, being prescribed by nursing staff.

Kay and Martin ${ }^{6}$ reported four cases of heel ischaemia in diabetics after application of graduated compression stockings. Callum ${ }^{7}$ and Johnson ${ }^{8}$ have also suggested that ischaemic complications are more frequent than previously thought. We wish to report two cases of ischaemic leg lesions, both of which appear to be associated with the use

Correspondence: N. Merrett, F.R.A.C.S., Prince Henry Hospital, Sydney, N.S.W., Australia.

Accepted: 20 July 1992 of graduated compression stockings in the treatment of DVT.

The graduated compression stockings used in̊ these cases were Thrombo Embolic Deterrent Stockings (TED), Kendall, Australia.

\section{Case 1}

A 77 year old man with a poor command of English was admitted with bilateral deep venous thrombosis extending to the pelvic veins, proven on venography. He was anticoagulated. TED stockings were fitted. He had a past history of metastatic carcinoma of the lung. There was no significant history of vascular disease.

After discharge, he spent most of his day sitting with his legs in a dependent position. He noticed that the stockings felt tight and were beginning to give him pain. He believed that this demonstrated that the stockings were working properly, and that their effect would be maximized if they were kept on at all times. As he was taking strong analgesics for his metastatic tumour, he minimized his discomfort. After 4 days, the legs became numb, and the discomfort settled.

Two weeks later, the patient presented to the casualty department where the stockings were removed (Figure 1). He had gangrenous areas on both feet and mottling of the thigh and calf. Femoral and popliteal pulses were present. He was admitted and the ischaemic areas were allowed to demarcate. The mottled appearance resolved, but he suffered ischaemic necrosis of the right forefoot 


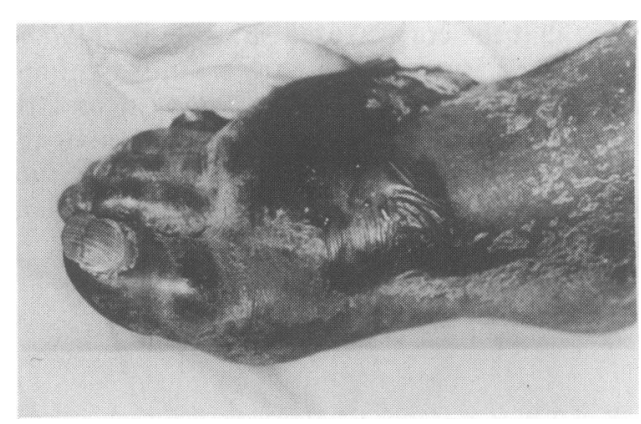

Figure 1 Bulla and mottling of feet associated with compression stockings in case 1 .

and several of the left toes. Three days after admission, he suffered a large intracerebral haemorrhage and died.

\section{Case 2}

A 39 year old man was admitted with a left cerebrovascular accident. On admission it was noted that his right pedal and popliteal pulses were absent. His past history included investigation for right leg claudication 6 years previously at another hospital, after which a sympathectomy had been performed. This had resolved his claudication symptoms.

Two weeks after admission, he developed a right deep venous thrombosis, proven on venography. He was commenced on anticoagulants and TED stockings were fitted. Three days later the patient developed an ulcer over the right lateral malleolus. The stockings were removed and the ulcer treated with dressings until it healed.

When the patient's dysphasia improved, a history was obtained that soon after the application of the stockings the patient noted that they felt tight, and he developed marked discomfort over the lateral malleolus. Owing to his dysphasia he was unable to communicate this discomfort to the nursing staff, who misinterpreted his attempts at communication.

\section{Discussion}

The effect on local blood flow of compressive or constrictive clothing was originally studied by the armed services when constricting clothes, shoes and gloves were found to be of importance in the development of frostbite and trenchfoot. These studies utilized volunteers from the armed forces with no evidence of peripheral vascular disease, and measured skin temperature, arterial and venous blood gas differences, and phlethysmography to quantify cutaneous blood flow. They demonstrated that even a small amount of external pressure could decrease blood flow considerably. It was noted that a pressure of $10 \mathrm{mmHg}$ produced a $10 \%$ reduction in cutaneous blood flow; $30 \mathrm{mmHg}$ a $25 \%$ drop, and $60 \mathrm{mmHg}$ an $84 \%$ drop. ${ }^{9}$ This demonstrated that not only did cutaneous pressure reduce local blood flow, but that the amount of reduction was proportional to the pressure being exerted. Thus any factor that increased this pressure could lower local blood flow far more than anticipated and possibly cause vascular compromise.

Graduated compression stockings are fitted by the patients' leg girth being measured to attain a particular pressure profile. This profile may be altered however by subsequent changes in leg girth. This pressure profile was studied by Horner. ${ }^{10} \mathrm{He}$ demonstrated that significant changes in the amount of pressure exerted by the stockings occur, related to changes in leg girth. Indeed an increase in circumference of $5 \mathrm{~cm}$ could double the amount of pressure being applied. This indicates that patients suffering from conditions associated with leg swelling, such as DVT or cardial failure, will experience sizeable increases in the pressure being exerted by the stockings if any swelling occurs after fitting. This may result in a significant reduction in local blood flow.

These factors of a drop in local blood flow and the potential of a higher than expected pressure profile suggests that in patients with peripheral vascular disease, graduated compression stockings have the potential to impair local blood flow to such an extent that ischaemia and ulceration may occur. Such a mechanism may have been responsible for the two cases that we have described.

The potential ischaemic problems have been recognized in the past. In the 1950s, Wilkens advised against elastic stockings in the treatment of venous disease if the pedal pulses were absent. ${ }^{11}$ It is also acknowledged by the manufacturers of the stockings who caution against their use in patients with severe atherosclerosis.

In spite of these warnings, it is generally thought that the complications of graduated compression stockings are benign and rare. ${ }^{2,4}$ Nonetheless, Kay and Martin ${ }^{6}$ described four cases of heel ischaemia from a single unit. Callum et al. ${ }^{7}$ surveying all 154 general surgeons in Scotland, found that $11 \%$ had noted ulcers or necrosis secondary to graduated compression stockings, with 38 cases recorded over a 5 year period.

Several considerations arise from these points. Patients may be at a far greater risk of ischaemic complications from these stockings than has been appreciated, and ischaemic lesions are far more common than has generally been accepted in the past. The risk of ischaemia is even greater if the 
patient suffers from a condition where there may be further swelling of the leg after fitting of the stocking.

This suggests that if a patient is likely to develop leg swelling, and particularly in patients with diabetes or peripheral vascular disease, then the patient should be measured over several days to ensure that the correct-sized stocking is obtained. Should the patient develop any pain or tightness, then the stockings should be removed and the patient remeasured. The ischaemic risks may also call into question any policy of routine prescription of graduated compression stockings, particularly by non-medically trained personnel.

\title{
References
}

1. Allan, A., Williams, J.T., Bolton, J.P. \& Le Quesne, L.P. The use of graduated compression stockings in the prevention of post operative deep vein thrombosis. Br J Surg 1983, 70: $172-175$.

2. Holford, C.P. Graded compression for preventing deep venous thrombosis. Br Med J 1976, 2: 969-971.

3. Scurr, J.H., Ibrahim, S.Z., Faber, R.G. \& Le Quesne, L.P. The efficacy of graduated compression stockings in the prevention of deep venous thrombosis. Br J Surg 1977, 64: 371-373.

4. Fasting, H., Anderson, K., Kraemer Nielson, H., Husted, S.E., Koopmann, H.D. \& Simonsen, O. Prevention of deep venous thrombosis. Acta Chir Scand 1985, 151: 245-248.

5. Bergqvist, D. \& Lindblad, B. It is possible to potentiate the thromboprophylactic effect of dextran with elastic compression stockings? Thromb Haemost 1983, 50 (Suppl 1): 247.

6. Kay, T.W.H. \& Martin, F.I. Heel ulcers in patients with long standing diabetes who wear antiembolism stockings. Med $J$ Austral 1986, 145: 290-293.

7. Callum, M.J., Ruckley, C.V., Dale, J.J. \& Harper, D.R. Hazards of compression treatment of the leg: an estimate from Scottish surgeons. $\mathrm{Br}$ Med J 1987, 295: 1382-1383.

8. Johnson, G.V. Elastic stockings. Br Med J 1988, 296: 720.

9. Halperin, M.H., Friedland, G.K. \& Wilkins, R.W. Effect of local compression upon blood flow in the extremities of man. Am Heart J 1948, 35: 221-227.

10. Horner, J., Lowth, L.C. \& Nicolaides, A.N. A pressure profile for elastic stockings. Br Med J 1980, 280: 818-821.

11. Wilkins, R.W., Mixter, G. \& Stanton, J.R. Elastic stockings in the prevention of pulmonary embolism. $N$ Engl J Med $\mathrm{O}$ 1953, 248: $1087-1090$

\section{Portal vein thrombosis due to Candida albicans associated with hepatic cirrhosis}

\author{
G. Torres, L.A. Gil Grande', B. Boixeda ${ }^{1}$, C. Martín-de-Argila, R. Barcena ${ }^{1}$ \\ and F. Garcia Hoz
}

Gastroenterology Department, Hospital 'Ramón y Cajal', 28034 Madrid, and 'Department of Medicine, Medical School of the University of Alcalá de Henares, 28800 Alcalá de Henares, Spain

\begin{abstract}
Summary: A case of portal vein thrombosis due to Candida albicans in a patient with alcoholic hepatic cirrhosis in the absence of hepatocarcinoma is described. Infection is a known cause of portal vein thrombosis but thrombosis by Candida albicans has not to our knowledge been previously reported.
\end{abstract}

\section{Introduction}

Portal vein thrombosis (PVT) is an uncommon complication of hepatic cirrhosis, with an incidence of $0.6 \%{ }^{1}$ The most frequent aetiology is invasion

Correspondence: C. Martín-de-Argila, M.D., C/Concha Espina 5, 28016 Madrid, Spain.

Accepted: 7 September 1992 from an associated hepatocellular carcinoma. ${ }^{2} \mathrm{We}$ present a patient with alcoholic hepatic cirrhosis who developed a portal vein thrombosis due to Candida albicans. Though infection is a known cause of PVT, it is rare and portal vein thrombosis by $C$. albicans has not to our knowledge been previously reported. 